\title{
Desarrollo de factores protectores y obstaculizadores de la resiliencia socioeducativa de niños y niñas, según la perspectiva docente
}

\section{Development of Protective and Hampering Factors of Socio-educational Resilience of Children, according to theTeaching Perspective}

\section{Desenvolvimento de fatores protetores e dificultadores}

\section{da resiliência socioeducativa de crianças, segundo a perspectiva do ensino}

\author{
Sonia Salvo-Garrido* (iD) orcid.org/0000-0002-9567-2285 \\ Susana San Martín-Praihuén* (iD) orcid.org/0000-0002-9704-811X \\ Joaquín Acuña-Cabrera*** (iD orcid.org/0000-0002-5407-3286 \\ Oscar Vivallo-Urra**** iD orcid.org/0000-0001-5200-0174
}

Para citar este artículo

Salvo-Garrido, S., San Martín-Praihuén, S., Acuña-Cabrera, J. y Vivallo-Urra, 0. (2021). Desarrollo de factores protectores y obstaculizadores de la resiliencia socioeducativa de niños y niñas, según la perspectiva docente. Revista Colombiana de Educación, 1(83), 1-18. https://doi.org/10.17227/rce.num83-11026.

Fecha de recepción: 25/12/2019

Fecha de evaluación: 07/09/2020

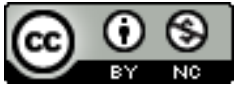

* Doctora en Estadística Multivariante. Profesora titular de la Universidad de la Frontera, Chile.

Correo: sonia.salvo@ufrontera.cl

* Magíster en Gerencia Social. Encargada de Asuntos Estudiantiles de la Dirección Académica de Postgrado, Universidad de la Frontera, Chile. Correo: susanasanmartin91@gmail.com

** Sociólogo. Asistente de proyectos de investigación en Laboratorio de Investigación Ciencias Sociales Aplicada, LICSA,

Temuco, Chile.. Correo: jeac924@gmail.com

*** Doctor En Ciencias Políticas por la Universidad Libre de Berlín, Alemania. Profesional de Apoyo en el área de Estudios y 


\section{Resumen}

Palabras clave: Resiliencia; escuelas públicas; maestros; logro académico; estudiantes en riesgo
Este artículo de investigación tiene como objetivo describir la percepción de docentes de alumnos vulnerables socioeconómica y psicosocialmente, con respecto de los factores protectores y obstaculizadores de la resiliencia académica y su contribución a los procesos resilientes de sus estudiantes, a través de elementos socioacadémicos, pedagógicos y de índole emocional/relacional. Mediante una metodología de tipo cualitativa, análisis de contenido, se abordó el contenido de 67 entrevistas realizadas a docentes pertenecientes a escuelas públicas y particulares subvencionadas vulnerables de Chile. Los principales resultados señalan la necesidad de que todos aquellos actores que conforman la comunidad educativa, en particular los profesores, afronten los retos de la educación en sus diferentes escenarios y desarrollen dinámicas que contribuyan, no solo al éxito académico, sino que también a la felicidad y al bienestar de los estudiantes. Desde esta perspectiva, relaciones positivas o negativas establecidas con los alumnos posibilitan u obstaculizan la capacidad de superar situaciones adversas, en términos de promover la resiliencia socioeducativa de la población más vulnerable del país.
Keywords: Resilience; public school; teachers; academic achievement; students at risk

Palavras-chave: resiliência; escolas públicas;

professores; conquistas

acadêmicas; alunos em risco

\section{Abstract}

This research article aims to describe the perception of teachers of socioeconomical and psychosocial vulnerable students, regarding the protective and hampering factors of academic resilience and their contribution to the resilient processes of their students, through socio-academic, pedagogical and emotional/relational elements. Through a qualitative methodology, content analysis, the content of 67 interviews conducted with teachers belonging to public schools and vulnerable subsidized individuals in Chile was addressed. The main results point to the need for all those who make up the educational community and, teachers particularly, to face the challenges of education in their different scenarios and to develop dynamics that contribute not only to academic success, but also to the happiness and well-being of students. From this perspective, the good relations established with the students make it possible to overcome adverse situations to them, being able to promote the socio-educational resilience of the most vulnerable population in the country.

\section{Resumo}

Este artigo de pesquisa tem como objetivo descrever a percepção de alguns professores de estudantes em situação de vulnerabilidade socioeconômica e psicossocial, respeito dos fatores protetores e dificultadores da resiliência acadêmica e sua contribuição para os processos resilientes de seus estudantes, por meio de elementos sócio acadêmicos, pedagógicos, assim como emocional/relacional. Através de uma metodologia qualitativa, a análise de conteúdo foi abordada mediante 67 entrevistas feitas a professores pertencentes a escolas públicas e particulares subvencionadas vulneráveis no Chile. Os principais resultados mostram a necessidade de que todos os atores que compõem a comunidade educativa, particularmente os professores, possam enfrentar os desafios da educação em seus diferentes cenários, desenvolvendo dinâmicas que contribuam, não só para o sucesso acadêmico, mas também para a felicidade e bem-estar dos alunos. Nessa perspectiva, as relações positivas ou negativas estabelecidas com os estudantes, possibilitam ou dificultam a capacidade de superar situações adversas, em termos de promover a resiliência socioeducativa da população mais vulnerável do país. 


\section{Introducción}

En la actualidad, indagar sobre los posibles determinantes del éxito académico de un niño o niña constituye una de las principales metas de la investigación educacional (Bravo-Sanzana et al., 2017; Cordero et al., 2015). Se destaca la situación de estudiantes denominados "resilientes", caracterizados por tener la capacidad de superar eventos o condiciones sociales adversas y de tener éxito académico exitoso, a pesar de las circunstancias negativas ambientales (Alivernini et al., 2016; Becoña, 2006; Forés, 2014; Fullana, 1998; Howard y Johnson, 2000; Sandoval-Hernández y Białowolski, 2016; Velásquez y Aguayo, 2011; Wu et al., 2013). Además, se ha planteado que cuando frente a eventos o condiciones adversas no se produce un desarrollo evolutivo adecuado, se ven afectadas las competencias, la adaptación social y los aprendizajes escolares (Muñoz y De Pedro Sotelo, 2005).

Asimismo, el actual sistema educacional chileno se vincula principalmente con un modelo educativo basado en competencias (Martínez-abad et al., 2017), con cuerpos jurídicos, como la Ley de Subvención Escolar Preferencial (SEP), que buscan mejorar con financiamiento fiscal la calidad de las escuelas públicas y de aquellas privadas con subvención estatal. Este financiamiento proporcionaría recursos financieros adicionales por cada alumno socialmente "prioritario" (proveniente de familias de bajos ingresos), transparentando a la comunidad los resultados de las escuelas y definiendo sus metas académicas (Shirley et al., 2013). En este contexto, se postula a la educación como un mecanismo valioso de justicia, movilidad e igualdad social (Hidalgo et al., 2018).

Sin embargo, la perspectiva liberal considera a las escuelas responsables de sus resultados y con suficiente disponibilidad de recursos para alcanzar las metas deseadas, si los educadores muestran suficiente fuerza de voluntad y perseverancia (Shirley et al., 2013). Junto a ello, en el sistema escolar chileno se ha extendido la desigualdad y la segregación socioeducativa, de acuerdo con el origen social (Corti et al., 2016). Como consecuencia, las escuelas dedicarían más tiempo a materias evaluadas, como el rendimiento en pruebas estandarizadas (SandovalHernández y Białowolski, 2016), que a otros contenidos, promoviendo mayor uniformidad en el enfoque curricular y disminuyendo la atención a la diversidad socioeducativa (Shirley et al., 2013). Por tanto, la educación tendría una clara urgencia de reflexionar en torno a los factores que influyen en su misión, para que la escuela sea parte importante del microsistema del niño (Toland y Carrigan, 2011), para su integración y desarrollo.

En términos socioeconómicos, las condiciones desfavorables de muchos niños y niñas implican también problemas políticos, económicos y sociales que desembocan en una progresiva pobreza (Greco et al., 2007). Adicionalmente, circularía una serie de pérdidas personales, familiares y sociales, así como un incremento de las desigualdades y de la exclusión social, pérdida de recursos y oportunidades económicas, deserción escolar y violencia intrafamiliar, que aumentan el grado de vulnerabilidad social de los niños, niñas y de sus familias (Greco et al., 2007).

Sin embargo, esta capacidad de superación de la adversidad se refleja en un conjunto de cualidades personales del ser humano orientadas a sobreponerse de situaciones vitales complejas y agobiantes (Chacón et al., 2017; Ramírez-Granizo y Castro-Sánchez, 2018; Yu et al., 2011). Específicamente, existiría una cercana relación entre las emociones positivas y la resiliencia, ya que las emociones positivas tienen una clara implicación en las estrategias de regulación de las experiencias negativas, estresantes o adversas (Greco et al., 2007). Además, se ha planteado que las experiencias emocionales positivas generan una ampliación 
cognoscitiva y conductual, y propician un estilo de afrontamiento adaptativo que favorecen la construcción de recursos propios personales resilientes de aprendizaje (Semanchin et al., 2018).

Desde esta perspectiva, la resiliencia se va desarrollando a través del tiempo, estableciéndose una asociación recíproca entre individuo y entorno. En el caso de niños, niñas y jóvenes, la escuela es donde se desarrollaría un mayor nivel de resiliencia (Ramírez-Granizo y Castro-Sánchez, 2018). Particularmente, la figura del docente, como formador central de la resiliencia (Acevedo y Restrepo, 2012a; López, 2010; Pitzer y Skinner, 2016; Sointu et al., 2016; Spellman, 2015) constituiría un actor clave para prevenir, detectar o intervenir en casos de niños y niñas que están sufriendo alguna situación de riesgo o de vulneración de derechos (Muñoz y De Pedro Sotelo, 2005). Por tanto, en el nivel de la escuela, construir resiliencia requiere de profesores capaces de implicarse en un proyecto educativo compartido e integral (Spellman, 2015).

Identificar los factores protectores y obstaculizadores tiene como finalidad detectar disposiciones individuales, como el afrontamiento, actitud positiva, sentido del humor, empatía, flexibilidad, perseverancia, religiosidad y optimismo (Gaxiola et al., 2011). Además, se requiere ahondar en los elementos contextuales socioacadémicos promotores de la resiliencia en el aula para conocer las estrategias de intervención desde la mirada docente (Alivernini et al., 2016).

\section{Resiliencia socioeducativa: antecedentes y definición del concepto}

La resiliencia socioeducativa es un proceso que resignifica la relación escuela-comunidadfamilia, cuyo fin es el desarrollo óptimo del estudiante, a través de la colaboración de alianzas estratégicas. El concepto de resiliencia socioeducativa nacería de la perspectiva ecológica de Urie Bronfenbrenner (Gifre y Esteban, 2012) para comprender mejor el efecto de esta en el desarrollo del estudiante, midiendo variables como la graduación, asistencia y tasas de deserción. Los actores exitosos lograrían la "cohesión cultural", construyendo relaciones de colaboración que rodean a los estudiantes (Leonard, 2011), destacándose los cambios perdurables en el modo en que un individuo percibe su ambiente y se relaciona con él (Gifre y Esteban, 2012).

Las consideraciones de Bronfenbrenner acerca del entorno permitieron comprender de manera científica la progresión y acomodación mutua entre un ser humano activo, su desarrollo y las propiedades cambiantes de los entornos inmediatos. Este proceso estaría influenciado por relaciones establecidas entre estos entornos y contextos más amplios (Gifre y Esteban, 2012). Aquí surgirían los roles decisivos de la familia, comunidad y sociedad y de los sujetos profesionales, como promotores de los recursos que tiene cada persona para lograr la autogestión del desarrollo personal y social (Extremera et al., 2010). En tal sentido, el concepto de resiliencia ha evolucionado hacia una noción dinámica, interactiva y global del desarrollo humano y, sobre todo, de la adaptación social (Uriarte, 2005), con lo que se aproxima a un modelo ecológico-transaccional, donde los factores involucrados son de carácter familiar, individual y comunitario (García-Vesga y Domínguez-de la Ossa, 2013; Martín-Lagos, 2018; Salvo et al., 2017). 
Ahora bien, un factor obstaculizador refiere a situaciones contextuales y/o personales que incrementan la probabilidad de desarrollar problemas emocionales y conductuales (BravoSanzana et al., 2016; González-Arratia et al., 2012). Y, al contrario, los factores protectores se entienden como aquellos que disminuyen el impacto de las condiciones de vulnerabilidad o de riesgo y favorecen la resistencia al daño, actualizando la fuerza interna y externa de las personas.

Actualmente, el estudio de la resiliencia socioeducativa se ha posicionado a nivel mundial. El informe del Programme for International Student Assessment (PISA) es una medición que permite aproximarse a las dimensiones socioculturales, al distinguir entre los niveles individuo y escuela. De esta manera es posible observar el rendimiento académico y la influencia en él del entorno familiar y, en especial, de todo el contexto educativo (García-Aracil et al., 2016; Salvo-Garrido et al., 2019), pues el informe proporciona datos comparables que posibilitan a los países mejorar sus políticas y estrategias educacionales.

\section{Docencia y resiliencia socioeducativa}

Numerosas investigaciones destacan el papel de los maestros en la construcción de la resiliencia de alumnos procedentes de entornos desfavorecidos y de exclusión social (Acevedo y Restrepo, 2012b; Hershkovitz, 2018; López, 2010; Pitzer y Skinner, 2016; Sointu et al., 2016; Spellman, 2015). Específicamente, señalan que las relaciones interpersonales entre profesores y estudiantes serían factores clave para los desarrollos académico, social y emocional, e influenciarían aspectos psicosociales y de aprendizaje dentro y fuera del aula (Landa y Ramírez, 2018). También se han enfocado en el periodo de la adolescencia (Swanson et al., 2011), revisando la implicación del estudiante con la escuela y con sus maestros (Rodríguez-Fernández et al., 2016a, 2016b; Rodríguez-Fernández et al., 2018), en términos de la comprensión del comportamiento saludable y del buen rendimiento académico (Rodríguez-Fernández et al., 2018).

Aquí, en términos de los procesos de resiliencia socioeducativa, la autoeficacia docente aparece como un factor central asociado al desempeño de los profesores, a su calidad en la práctica educativa y enseñanza, y a los resultados de aprendizaje logrados por los estudiantes (Gil, 2016). Los profesores serían capaces de entregar aprendizajes que incluyen la consecución de objetivos y metas, ante los cuales estudiantes resilientes tienen capacidad de dar respuestas, a través del apoyo de su entorno y de ser agentes de sus propios cambios (Gordon, 2001; Landa y Ramírez, 2018; Reoyo et al., 2017).

La eficacia docente puede organizarse en cuatro áreas de factores protectores asociados al trabajo con niños y niñas resilientes: características afectivas; habilidades, como la creatividad y apertura a nuevos desafíos; técnicas de gestión del aula, y conocimientos académicos (Reoyo et al., 2017). También las expectativas positivas del docente acerca de la proyección académica del estudiante y de su capacidad para enfrentar las dificultades de la vida, constituyen factores protectores de la resiliencia socioeducativa (Dueñas, Godoy, Duarte, y López, 2019). Así, se revela la interacción profesor-alumno y el medio ambiente, en un proceso de co-construcción basada en la coordinación y sincronización de progresiones; el docente se constituye en facilitador del aprendizaje, por su disposición a desarrollarse, autodirigirse, reajustarse y promover la resiliencia (Aguaded y Almeida, 2016).

Desde esta perspectiva, este artículo procura describir la percepción de docentes de alumnos vulnerables socioeconómica y psicosocialmente, con respecto de los factores 
protectores y obstaculizadores de la resiliencia académica y su contribución a los procesos resilientes de sus estudiantes, a través de elementos socioacadémicos, pedagógicos y de índole emocional/relacional. La identificación de estos factores permitirá dar cuenta de oportunidades, experiencias, características y logros de los estudiantes de primer ciclo, con la finalidad de ser extrapolados a otros contextos socioeducativos similares

\section{Metodología}

Se utilizó un enfoque cualitativo para indagar en la dimensión subjetiva de los participantes (Vieytes, 2004), el estudio fue de corte transversal y no experimental. Para analizar los relatos de las entrevistas, se utilizó la técnica de análisis de contenido (Gerbic y Stacey, 2005). Es un procedimiento que permite analizar y cuantificar los materiales de la entrevista. Permitirá integrar la heterogeneidad de los actores participantes y relatos, y profundizar en los significados que los docentes atribuyen a los factores protectores y obstaculizadores de la resiliencia socioeducativa, desde la libertad subjetiva de los docentes entrevistados. Por último, inquirir cómo, desde las vivencias profesionales de estos significados e interpretaciones de la propia realidad profesional, se pueden proyectar contribuciones de factores protectores y obstaculizadores para mejorar el rendimiento académico de los estudiantes de Chile.

La muestra quedó conformada por 67 docentes, 45 mujeres y 22 hombres con experiencia profesional entre 3 y 42 años, que realizaban clases en el primer ciclo de enseñanza básica en establecimientos educacionales públicos y particulares subvencionados, que tuviesen la mayor cantidad de alumnos resilientes de los territorios comprendidos entre la región de La Araucanía y la región de Aysén, sur de Chile. Los alumnos resilientes se detectaron en función de un índice socioeconómico compuesto estandarizado, desarrollado por Salvo-Garrido et al. (2020), y del rendimiento académico obtenido por los alumnos de cuarto año de Enseñanza Básica en las pruebas Sistema de Medición de la Calidad de la Educación (Simce). Estudiantes con un valor del índice menor o igual a cero y con un rendimiento Simce promedio superior al nivel de aprendizaje adecuado de acuerdo con el Ministerio de Educación de Chile (Mineduc, 2012, 2013a, 2013b), fueron considerados resilientes.

Se realizaron entrevistas semiestructuradas (Hernández-Sampieri et al., 2010) en distintos momentos del periodo octubre, 2017-noviembre, 2018, las cuales presentaron una guía temática de los principales tópicos a abordar con los participantes, previo sometimiento al juicio de cinco expertos del área de estudio. Se profundizó en temáticas que emergieron espontáneamente del testimonio de los participantes, lo que posibilitó la emergencia de contenidos no contemplados por el equipo de investigación.

Para la aplicación de las entrevistas, se solicitó autorización a los directores de los establecimientos educacionales. Se realizaron reuniones informativas donde se explicaron los objetivos y alcances del estudio, para finalmente aplicar un consentimiento informado que resguardó los siguientes principios éticos: voluntariedad, autorización a grabar la entrevista, resguardo de la información, confidencialidad, además del anonimato de los participantes y de los datos específicos sobre los estudiantes o la escuela. Todos los protocolos fueron sometidos y validados por el Comité Ético Científico de la Universidad de La Frontera.

La codificación y clasificación de información se formuló con base en criterios establecidos por los investigadores, procurando un diálogo entre la literatura científica y la información 
empírica. De esta manera, emergieron constructos en un proceso analítico y sistemático (construcción de protocolo de codificación y unitización), que se fue modificando según el tipo de información recogida, para representar los constructos relevantes condensados y emergentes de las entrevistas (Schettini y Cortazzo, 2015). El análisis permitió integrar la heterogeneidad de los actores participantes y relatos, para profundizar en los significados en torno a factores protectores y obstaculizadores de la resiliencia, desde la libertad subjetiva de las personas entrevistadas (Hernández-Sampieri et al., 2010).

\section{Resultados}

Desde la perspectiva del profesor (como actor significativo para dinamizar la resiliencia socioeducativa), se identificaron sistemas y categorías de resiliencia socioeducativa, en cuatro ámbitos de análisis: convivencia escolar, afectividad, material educativo y articulación y espacios educativos.

\section{Características personales de estudiantes resilientes}

Considerando como aspectos importantes el bienestar y la calidad de vida (Crespo, 2016), los profesores señalaron que los niños y niñas resilientes desarrollan capacidades debido a la conjugación de resultados académicos mejores a lo esperado y de ciertas condiciones contextuales de riesgo. Según el reporte docente, la motivación propia de los estudiantes por adquirir conocimiento constituye una destacada herramienta de autoeficacia académica.

Los estudiantes de esas características, por ejemplo, siempre quieren aprender más, siempre buscan más tareas, son de esos estudiantes que... eh... lo averiguan todo o... cuando quedan con una duda, al día siguiente, por ejemplo, uno les puede decir "ya, averígüenme" y al día siguiente "iieh, tía!!"... "¿y lo averiguó, de qué se trata?"... eh... siempre están buscando libros, leyendo".

También se señaló el temple que presentan los estudiantes resilientes como forma de enfrentar dificultades a través del esfuerzo personal. Aquí destacan la capacidad de mantener la calma ante los problemas y un foco en encontrar soluciones, todo ello con el apoyo social contextual requerido para el soporte de una actitud resiliente.

Son alumnos que son muy esforzados, que tienen alta tolerancia a la frustración, son capaces de detectar las cosas que puedan mejorar ellos y buscan, además, las herramientas que están más a mano y siempre buscan. Yo tengo la visión de que todos los niños y las personas pueden ser resilientes, pero depende un poco del entorno también, porque si yo quiero salir adelante, en el caso de los niños, si no tengo apoyo o de adonde agárrame o a quien recurres, es más difícil a veces.

Según los profesores, los estudiantes resilientes serían personas esforzadas que se plantean metas y objetivos de superación personal y académica, que asumen actitudes positivas frente a problemas para lograr alcanzar estándares superiores de vida, en términos personales y socioeconómicos.

No... aquí va estar en el pueblo, si no que tienen otra visión más amplia, de que ellos pueden viajar "oh, que rico viajar en avión"... Entonces, ellos... como que esto les ayuda a ellos a superarse también como personas, no ha quedarse aquí, nomás, sino que ser alguien más en la vida... decir "chuta, si yo en realidad más grande... eh... a futuro quiero ser esto porque me voy a poder dar ciertos lujos"... que en el fondo lo van a ayudar a superarse como personas y a tener otro tipo de... de superar otros tipos de vida, superarse en la vida y no a quedarse aquí no más. 


\section{Factores protectores de la resiliencia socioeducativa, desde la perspectiva docente}

En el ámbito de "convivencia escolar (micro y meso sistemas)", se destacan los factores de "resguardo y la acogida" (38 \% de las citas). Luego, aparece la "disciplina" (22 \%), "buena convivencia" (11\%) y "compañerismo" (10 \%), principalmente observados con respecto a la escuela, el trabajo colaborativo dentro de la escuela y entre el profesor y otros actores significativos. Otros factores, como la seguridad, sentido de pertenencia, cooperación, solidaridad y respeto, se distribuyen en pequeños porcentajes.

El reporte docente enfatizó estos niveles relacionales en la comunidad educativa, junto a un conjunto de interacciones positivas de parte de figuras adultas hacia los niños y niñas, que generaron espacios de desarrollo y vínculos afectivos compensatorios de contextos familiares problemáticos o disfuncionales.

las mismas relaciones que tengan con las personas, con las relaciones que tengan con el inspector, que tengan con el auxiliar [...]. Porque uno igual va aprendiendo de... de ellos o el consejo que te puede dar él... no sé, el auxiliar, o que el mismo auxiliar te reciba con cariño, que conozca tu nombre. Entonces, uno igual se siente importante, se siente querido... eh... y eso se da mucho aquí, por lo menos yo lo veo que...

Asimismo, el apoyo docente, en términos de acogida y consideración por la persona del estudiante, puede configurar espacios y climas protectores y de confianza que estimulan los procesos de resiliencia socioeducativa.

Sí, tenemos lo... también tenemos casos, obviamente, de... de niños que quieren salir adelante a pesar de su adversidad y que la familia obviamente no apoya. Pero, ellos buscan su segunda casa, porque aquí el colegio es súper familiar. Entonces, el colegio pasa a ser su segunda casa y ellos son muy cercanos a su profesor jefe o al profesor que tiene más afinidad. Entonces, ellos son de contar sus problemas, de contar lo que está pasando... "Profe, ¿cómo lo puedo hacer? ¿Cómo lo puedo conseguir? Me falta esto, me falta lo otro... eh... no entendí esta materia, no entendí esto otro". Y ellos se sienten de repente con mucha más confianza con los profesores en este caso de estos niños, que, con sus propias familias, contándote de hasta de lo mínimo que ocurre en su casa.

En el ámbito "afectivo", se destacó mayoritariamente el "sistema micro profesor" (85 \%), lo que muestra la importancia del actor profesor como agente significativo para dinamizar, desde el plano de la afectividad, los procesos resilientes de los niños y niñas. Aquí aparecen las categorías de compromiso y responsabilidad del profesor hacia los estudiantes, además de la confianza, motivación, orientación, presencia y apoyo, cariño y las actitudes protectoras. Estos factores promoverían la resiliencia y el buen rendimiento académico, mediante lazos de confianza que permiten una atención personalizada y un foco en el bienestar socioemocional del estudiante. De manera específica, se señaló un proceso de acogida que contendría afectivamente al niño, promoviendo la conexión emocional con los tópicos curriculares y un bienestar socioafectivo que reforzaría el interés del estudiante por las actividades pedagógicas. 
[...] sí, yo gracias a Dios puedo decir que logro encantar a los alumnos con la asignatura, que es lo principal. Mi política es que siempre, es que a la buena se consiguen más cosas que a la mala. Entonces, lo primero que yo hago es encantarles con la asignatura y después empezar a sacar provecho para que sus sueños se vayan haciendo realidad.

[...] y como te digo yo... y los chicos... yo creo que lo que más les gusta de este colegio es la capacidad que tenemos los profesores por... eh... ¿"apapacharlos" ?, por decirlo de alguna manera. Porque yo, por ejemplo, como profesora de matemáticas, puedo ser muy mañosa, pero también con ellos trato de ser bien humana, porque siento que son niños que necesitan cariño, necesitan afecto, necesitan que los entiendan.

Otro factor protector señalado son las diferentes estrategias educativas, entre las que se destacan las "metodologías de enseñanza-aprendizaje, E-A" (34\%), estas constituyen métodos que estimulan el proceso de E-A y el desempeño académico, y proveen herramientas que refuerzan tanto habilidades blandas como otras habilidades útiles para un desarrollo integral y de vida y el "refuerzo positivo-premiación" (31 \%), definido como comunicaciones positivas destinadas a dinamizar al estudiante académicamente, con foco en su bienestar emocional. La premiación constituiría un método de refuerzo para destacar y afianzar características positivas de los alumnos, tanto personales como académicas.

Claro y hacemos mucho. Por ejemplo, este año hicimos ferias científicas, exposiciones artísticas [...] ¿Ya? Eh... hacemos, hace poquito hicimos una gala abierta a la comunidad, en donde los niños se lucen y muestran toda su... la parte artística. Entonces, estamos siempre pensando en hacer muchas actividades, mucha integración con los chicos.

El esfuerzo... el esfuerzo y la constancia... eh... no sé si algún otro profesor comentó que acá se hacen dos premiaciones al año [...]. Claro, el primer semestre se hace una que es el mejor compañero, que lo eligen los chicos, el alumno integral, y... eh... mejor rendimiento. Entonces, dentro de ese alumno integral, se busca de repente rescatar a ese niño que vive y tiene resiliencia de acuerdo a su entorno [...] y, por ejemplo, en el segundo semestre, que la primera elección la hizo la otra profesora, la segunda la hice yo.

En el ámbito "articulación y espacios educativos que vienen desde la escuela", se representa al establecimiento educacional como factor protector encargado de articular diferentes dinámicas en que aparecen explícitos elementos de formación y de convivencia, en torno al ambiente educativo. Específicamente, se destacan las redes internas conformadas en la institución escolar (52 \%), también el apoyo escolar mediante la generación de redes de apoyo internas (30\%). Aquí se alude a la promoción de acciones protectoras, además de la red interna económica (22 \%), definida como el apoyo económico al estudiante, en cuanto a útiles escolares, ropa y otros elementos que pueda necesitar. Finalmente, aparecen como factores protectores el trabajo colaborativo (22\%) y las actividades extracurriculares, salidas pedagógicas y actividades deportivas (18\%).

De esta manera, emergerían como factores protectores las interacciones y vínculos que los estudiantes experimentarían con algunas figuras adultas que componen la comunidad educativa, lo que generaría flujos solidarios de índole material y socioemocional. En estas redes internas surgirían espacios de confianza y de apoyo para abordar las dificultades que presentan los estudiantes y expandir sus expectativas u horizontes de vida hacia trayectorias educativas superiores.

en cambio, aquí los niños tienen mucha cercanía con nosotros... ya tenemos un ambiente y se genera un vínculo con ellos y a nivel de todos, no solo con los profesores. Ellos saben que pueden recurrir a la psicóloga, secretario, portero, auxiliar y toda esa información se encauza para que ellos puedan salir adelante. Como te decía, son niños muy esforzados muy responsables, niños 
que a veces... a ver, nosotros también usamos criterios con estos niños, porque sabemos las dificultades que ellos tienen; eso los marca, muy capaces de buscar ayuda.

Si hay una carencia, por ejemplo, de un uniforme, de materiales, entre los colegas por ahí hacemos un [...] una búsqueda de cositas y le ayudamos. Entonces, siempre estamos tratando de sacar adelante a los niños y... y en Octavo, por ejemplo, nos preocupamos de estos niños que, pudieran tener una proyección... eh... de [...] abandono finalmente del estudio. Entonces, nos preocupamos de ellos para que durante el año egresen de octavo, pero que sigan en el mismo colegio. Una de las intenciones del sostenedor de abrir la enseñanza básica, a pasar a educación media, es que siguiéramos teniendo a estos alumnos y con proyecciones académicas no técnico profesional, que pensábamos nosotros que, por estar inserto, se podía dar esa [...] esa temática, pero finalmente [...] creo que fue la buena opción que nuestro horizonte siempre estar pensando en que nuestros alumnos son capaces de sacar una carrera universitaria.

\section{Factores obstaculizadores para la resiliencia socioeducativa, desde la perspectiva docente}

En el ámbito de "convivencia escolar", se destacan factores obstaculizadores. El más mencionado fue el clima escolar (43\%), que alude a la violencia, bullying, falta de normas o disciplina, principalmente en espacios educativos como la escuela y salas de clases. Asimismo, aparecen como factores perjudiciales el "aburrimiento", "desmotivación", "irresponsabilidad" o "influencias negativas" (43\%), que afectando al aula como espacio significativo y también al actor docente. Finalmente, aparecen las "drogas y alcohol" (13\%), como factores negativos latentes en la escuela.

Por otra parte, los profesores reportan que estos factores se entrecruzan con condiciones familiares de vulnerabilidad. Aquí sería central un estilo docente de liderazgo no autoritario, con proximidad afectiva y motivadora, que exprese un respeto explícito hacia las personas, valore sus capacidades y acoja sus inquietudes, características que Salvo-Garrido y CisternasSalcedo (2020) denominaron líder coach-maestro.

A ver... también los profesores jugamos [sic] un rol bastante importante en cuanto a lo que es el desarrollo positivo o negativo en cuanto a los niños, porque, por ejemplo, si el chico entra a un ambiente hostil, a un ambiente donde el profesor es como muy tirano o la profesora piensa que se las sabe todas y no les da instancia a los chicos, qué se yo... obviamente, el niño no va a rendir lo que tiene que rendir, porque ellos son niños de partida, piensan que haciéndole la contraria al profesor a la profesora ellos se están beneficiando ¿Me entiende o no? Entonces, de partida el ambiente donde ellos llegan... porque muchos llegan con problemas de la casa y, más encima, que llegan a una sala donde el profesor profesora los sube y los baja por cualquier motivo o los desalienta rápidamente. $O$ si el niño tiene una respuesta y la respuesta era incorrecta y el profesor lo ridiculiza, es obvio que el niño jamás va a querer participar y aparte de eso se va a volver contrario al profe. Y no, solamente, va a repercutir en él, sino que también va a influenciar a otro y esta cosa se vuelve como una cadena. Entonces, igual es importante eso, el clima de la sala de clases y el clima entre ellos se supone que acá en este colegio enseñamos valores. Entonces, obviamente, tú tienes que empezar por el respeto contigo para proyectárselo a los demás, enseñarles el respeto entre ellos, la convivencia, etcétera. Hay un montón de cuestiones ahí.

En el ámbito "afectivo" destacan las ausencias y desinterés que puede presentar el profesor hacia el alumno, desde el micro y meso sistema. Específicamente, se señala la no interferencia, el desconocimiento, el no potenciar al estudiante, la carencia de comunicación 
y perdida de vínculos afectivos. Aquí los docentes entrevistados identificaron la carencia de comunicación (31\%) y la no interferencia en las problemáticas o dificultades del menor (35\%), como los obstaculizadores más latentes para un niño resiliente. Cabe señalar que estas categorías desfavorables refieren a un déficit de confianza en el profesor, de parte de la generación actual de estudiantes, en contraste con aquellos vínculos afectivos más próximos y de mayor apertura que se habrían observado en generaciones anteriores.

Falta esa confianza que tienen que... no sé si la perdieron, porque yo me acuerdo... como tengo hartos años, antes los niños eran más abiertos, le contaban a los profesores... "sabe que mi mamá se fue de la casa" o "mi papá se fue de la casa y ahora tengo otro papá u otra mamá". Antes eran más abiertos; ahora no cuenta nada... más para adentro.

En el ámbito "material y físico" se destaca el microsistema del estudiante, quien se enfrenta con los riesgos de forma directa en sus espacios y ambientes más próximos. Los obstaculizadores presentes dentro del aula serían los más relevantes (50\%), se destacan el hacinamiento y acústica de la sala escolar, además de la influencia distractora de las TIC dentro del aula. Además, el reporte docente refiere que las condiciones deficientes del mobiliario y de las salas de clases influirían negativamente en la experiencia del niño en el aula.

Yo creo que más que el que no tengamos buen inmobiliario, o las salas que estén un poco destruidas... bueno, igual influye... por supuesto que va a influir en cuanto a cómo se siente un niño dentro de la sala, porque obviamente es más lindo llegar donde esté bonito, limpiecito, abrigadito, pero no creo que sea un factor tan incipiente eso.

Otro factor obstaculizador sería la dependencia socioafectiva, que proviene de un acostumbramiento vincular del alumno respecto de algún elemento docente protector. Específicamente, el mismo sistema de distribución de los cursos a los profesores puede afectar el desarrollo de vínculos protectores docente-alumno. La relación socioafectiva de confianza con el profesor se vería interrumpida por la relocalización de los profesores en otros cursos, pues se pierde la continuidad del vínculo desarrollado con el alumno. Asimismo, la interrupción no deseada de este vínculo afectivo afectaría negativamente el proceso educativo y resiliente del estudiante.

La motivación del chico cuando empieza eso a ver... A veces sucede que nosotros... bueno todos los años a ti te dan un horario que no es el mismo. Entonces a veces tú no sigues con los cursos; estás un año con ellos, lograrás una cierta comunicación, pero después viene otro y es empezar de nuevo ahí. El chiquillo se cae, porque no tiene la confianza, porque a lo mejor el profesor que vino antes llegó a ellos, ellos sintieron que él era su apoyo y el empezar de nuevo, cada vez el empezar de nuevo, eso cansa a los chiquillos y volvemos a cero y ellos vuelven a cero rápidamente, porque su autoestima es... no es pareja su autoestima, sino que tiene vacíos. Entonces, si tiene el apoyo del profesor que lo conoce, lo va a seguir. Pero, si él empieza de nuevo, ahí ya... como ya empieza a decantar y dice no, no puedo. Y los chiquillos son muy fáciles a frustrarse. El joven, si no logra hoy día los frutos rápidos, se frustra.

En el ámbito "educativo", el factor obstaculizador predominante fue la inadecuación pedagógica y vincular con respecto de la diversidad estudiantil, además de una inadecuada gestión (71\%). Esta última, asociada a dificultades para atender - con pertinencia- la diversidad de necesidades estudiantiles del establecimiento; se destaca el nivel del profesor como actor significativo, donde este obstaculizador se hace más presente.

son cuestiones que a mí nunca me la enseñaron en la universidad jamás. Entonces, sí se hace el acompañamiento yo doy fe de eso, porque yo lo hago diariamente o constantemente y, por lo tanto, es necesario, es necesario. En general, se entiende el nivel de educación, el grado de profesionalidad que tenga la persona que está trabajando con este tipo de alumnos. Va a 
depender de eso y los papás se tienen que comprometer. Ellos entienden que esto es una ayuda, no una molestia. Claro, nosotros trabajamos sobre la base de ciertas limitaciones. Ya hay cuestiones que son familiares y que uno no puede entrar, no se puede interferir... que uno... lo lamento por supuesto, pero aquí ya son tres instituciones del Estado que están con esa familia. Por lo tanto, uno hace un poquito más de lo necesario. En términos de esos límites no se puede hacer mucho.

Específicamente, los profesores aluden a la falta de preparación docente de origen, en términos de disponer de estrategias de apoyo para contribuir a la superación de situaciones adversas de los estudiantes. También, la vinculación docente con la familia constituiría un área difícil de coordinar, limita las posibilidades de actuación docente en apoyo a los procesos de resiliencia socioeducativa.

\section{Discusión y conclusiones}

Los resultados permiten plantear que las relaciones de los profesores y el entorno educativo son determinantes para el desarrollo u obstrucción de los procesos de resiliencia socioeducativa, especialmente en los niveles internos e inmediatos de contribución al desarrollo integral del estudiante (Gifre y Esteban, 2012). En tal sentido, tal como señalan Rodríguez-Fernández et al. (2018), los procesos de resiliencia socioeducativa se refuerzan cuando se establecen relaciones docente-alumno positivas, lo cual se vería expresado en un buen rendimiento académico de los estudiantes. Específicamente, las relaciones basadas en la acogida, orientación, cariño, metodologías de enseñanza aprendizaje, redes de apoyo, entre otras, combinadas con elementos socioacadémicos y pedagógicos, contribuirían a promover la figura del docente como promotor central de la resiliencia socioeducativa (Acevedo y Restrepo, 2012b; López, 2010; Pitzer y Skinner, 2016; Sointu et al., 2016; Spellman, 2015). Por el contrario, las relaciones basadas en una deficiente convivencia escolar, carencia de comunicación entre profesor y estudiante, junto a una inadecuación del modelo educacional a la diversidad estudiantil, contribuirían al retroceso de los procesos resilientes (Muñoz y De Pedro Sotelo, 2005), en aquellos contextos vulnerables y desiguales de donde provienen los estudiantes (Corti et al., 2016).

Con relación a la identificación de factores protectores y obstaculizadores, se observó que presentan una estrecha relación con la literatura científica que señala que unas adecuadas prácticas docentes mejoran la calidad de enseñanza de los estudiantes (Muñoz y De Pedro Sotelo, 2005; Reoyo et al., 2017). Asimismo, resalta la capacidad del niño para observar e incorporarse a patrones en uso de actividad progresivamente más compleja, bajo la guía y el apoyo educativo de personas que poseen conocimientos y/o destrezas todavía no adquiridas por el niño, y con las cuales se ha establecido una relación emocional positiva (Gifre y Esteban, 2012).También, es crucial atender y ampliar en el niño la disponibilidad de oportunidades, recursos y estímulos, para implicarse en las actividades que ha aprendido en contextos anteriores, con la intervención activa o la guía adulta directa y colaborativa (Leonard, 2011).

En tal sentido, considerando la resiliencia socioeducativa en su acepción general, los factores protectores se considerarían como un proceso de transformación en la convivencia entre padres, profesores y alumnos (García-Vesga y Domínguez-de la Ossa, 2013; Gifre y Esteban, 2012; Martín-Lagos, 2018). El foco del proceso serían factores socioacadémicos, pedagógicos y emocionales, que pueden favorecer de manera gradual al niño, contribuyen a su desempeño académico y al desarrollo de conductas resilientes ante la adversidad (Amar, Kotliarenko, y Abello, 2003). Además, los factores protectores socioafectivos son los que obtuvieron mayor preponderancia en el microsistema-profesor, presentando una mayor concurrencia con la teoría (Reoyo et al., 2017). Aquí se trata de la capacidad docente para 
suplir afectivamente la ausencia de padres o familiares cercanos, la resiliencia se constituye también en una cualidad psicológica que ayuda a la adaptación positiva ante procesos o periodos vitales o historiográficos adversos (Chacón et al., 2017; Yu et al., 2011). Esto se explica gracias a la mediación psicológica entre el estímulo ambiental y la conducta con la que responde una persona, y se traduce en el modo en que esta vivencia una situación vital (Bronfenbrenner, 1987).

Es aquí donde se sugieren metodologías de trabajo didácticas, aula interactiva y dinámicas que, en complemento con lo planteado por Gil (2016), establecen el rol del profesor como un guía que motiva e inspira confianza dentro del aula, en lugar de un expositor de contenido (Aguaded y Almeida, 2016). El docente proporcionaría apoyo y motivación a cada estudiante, quien aprende de sus compañeros, así como del profesor (Landa y Ramírez, 2018). Mantener una trayectoria resiliente depende en su gran mayoría del soporte y de la afectividad entregada al estudiante (Gifre y Esteban, 2012; Ramírez-Granizo y Castro-Sánchez, 2018). Por tanto, serían principalmente los profesores los encargados de formar a "sujetos preparados para afrontar con posibilidades reales de éxito las inevitables dificultades de la vida" (Turner, 2017). Y aquí, las expectativas docentes respecto de las posibilidades prospectivas del joven surgirían también como un factor protector relevante (Dueñas et al., 2019).

Respecto de los alcances de este estudio, identificar los factores protectores y obstaculizadores permite favorecer la resiliencia y expandirla a niños y niñas en situaciones complejas. Así, la relación establecida entre los sistemas socioecológicos podría disponer de derroteros estratégicos para el funcionamiento orientado a enfrentar las incertidumbres y los factores adversos generadores de estrés (Castillo-Villanueva y Velázquez-Torres, 2015). Y desde las políticas públicas, los factores protectores permiten proveer de oportunidades, estatus, recursos y estabilidad como elementos favorables al apoyo de adultos en actividades compartidas con los menores (García-Aracil et al., 2016; Gifre y Esteban, 2012; Salvo-Garrido et al., 2019).

Sin embargo, un desarrollo del sistema educativo basado en competencias y caracterizado por altos niveles de desigualdad (Corti et al., 2016) tiende a debilitar los procesos de resiliencia socioeducativa y a profundizar los factores obstaculizadores, lo que genera dificultades permanentes para el rendimiento del alumno y su desarrollo integral. Por tanto, los factores protectores debiesen contribuir a la eliminación de años de desventajas educativas, masificándose y replicándose, ya que la resiliencia puede ser intervenida y fomentada estratégicamente por la institucionalidad pública educacional y las comunidades educativas, para favorecer a miles de estudiantes provenientes de contextos vulnerables. 


\section{Referencias}

Acevedo, V. y Restrepo, L. (2012a). De profesores, familias y estudiantes: fortalecimiento de la resiliencia en la escuela. Revista Latinoamericana de Ciencias Sociales, Niñez y Juventud, 10, 301-319.

Acevedo, V. y Restrepo, L. (2012b). De profesores, familias y estudiantes: fortalecimiento de la resiliencia en la escuela. Revista Latinoamericana de Ciencias Sociales, Niñez y Juventud, 10, 301-319. https://dialnet.unirioja.es/servlet/articulo?codigo $=3935040$

Aguaded, M. y Almeida, N. (2016). La resiliencia del docente como factor crucial para superar las adversidades en una sociedad de cambios. Tendencias Pedagógicas, (28), 167-180.

Alivernini, F., Manganelli, S. y Lucidi, F. (2016). The last shall be the first: Competencies, equity and the power of resilience in the Italian school system. Learning and Individual Differences, 51, 19-28. https://doi.org/10.1016/j.lindif.2016.08.010

Amar, J., Kotliarenko, A. y Abello, R. (2003). Factores psicosociales asociados con la resiliencia en niños colombianos víctimas de violencia intrafamiliar. Investigación y Desarrollo, 11(1), 162-197.

Becoña, E. (2006). Resiliencia: definición, características y utilidad del concepto. Revista de $\begin{array}{lllll}\text { Psicopatología } \quad y \quad \text { Psicología } & \text { Clínica, } & \text { 125-146. }\end{array}$ http://aepcp.net/arc/01.2006(3).Becona.pdf

Bravo-Sanzana, M., Salvo-Garrido, S. y Mieres-Chacaltana, M. (2016). El clima social escolar en el logro de aprendizajes y el desarrollo integral, ¿cómo afecta a la salud mental de los niños y niñas? Salud Pública de México, 58(6). https://doi.org/10.21149/spm.v58i6.7913

Bravo-Sanzana, M., Salvo-Garrido, S., Mieres, M., Mansilla, J. y Hederich, C. (2017). Perfiles de desempeño académico: la importancia de las expectativas familiares. Perfiles Latinoamericanos, 25(50), 361-386. https://doi.org/10.18504/pl2550-016-2017

Bronfenbrenner, U. (1987). La ecología del desarrollo humano. Paidós.

Castillo-Villanueva, L. y Velázquez-Torres, D. (2015). Sistemas complejos adaptativos, sistemas socio-ecológicos y resiliencia. Quivera, 17(2), 11-32.

Chacón, R., Padial, R., Zurita, F., Castro, M., González, G. y Ramírez, I. (2017). Clima motivacional e inteligencia emocional en la promoción de hábitos saludables: una revisión narrativa. Revista Digital de Educación Física, 9(49), 108-117.

Cordero, J., Pedraja, F. y Simancas, R. (2015). Factores del éxito escolar en condiciones socioeconómicas desfavorables. Revista de Educación, (370), 172-198. https://doi.org/10.4438/1988-592X-RE-2015-370-302

Corti, A., Belén, C., y Luján, M. (2016). La diversificación educativa y la segmentación social. Signos de una época de nuevas desigualdades. Revista Colombiana de Educación, (70), 287-304. http://www.scielo.org.co/scielo.php?pid=S012039162016000100013\&script=sci_abstract\&tlng=es

Crespo, M. (2016). Resiliencia, bienestar y aprendizaje a lo largo de la vida. International Journal of Developmental and Educational Psychology, 1(2), 161-170. 
Dueñas, X., Godoy, S., Duarte, J. y López, C. (2019). La resiliencia en el logro educativo de los estudiantes colombianos. Revista Colombiana de Educación, 1(76), 69-90. https://doi.org/10.17227/rce.num76-8037

Extremera, N., Durán, A. y Rey, L. (2010). Recursos personales, síndrome de estar quemado por el trabajo y sintomatología asociada al estrés en docentes de enseñanza primaria y secundaria. Ansiedad y Estrés, 16(1), 47-60.

Forés, A. (2014). La resiliència, una aposta per canviar models d'intervenció. En No Col·lecció Infància i Adolescència (pp. 205-211). Generalitat de Catalunya.

Fullana, J. (1998). La búsqueda de factores protectores del fracaso escolar en niños en situación de riesgo mediante un estudio de casos. Revista de Investigación Educativa, RIE, 16(1), 47-70.

García-Aracil, A., Neira, I. y Albert, C. (2016). Influencia del capital social y cultural en el conocimiento financiero de los adolescentes: entorno familiar y escolar social. Revista de Educación, (374), 94-117. https://doi.org/10.4438/1988-592X-RE-2016-374-327

García-Vesga, M. y Domínguez-de la Ossa, E. (2013). Desarrollo teórico de la resiliencia y su aplicación en situaciones adversas: una revisión analítica. Revista Latinoamericana de Ciencias Sociales, Niñez y Juventud, 11(1), 63-77. https://doi.org/10.11600/1692715x.1113300812

Gaxiola, J., Frías, M., Hurtado, M., Salcido, L., y Figueroa, M. (2011). Validación del inventario de resiliencia (IRES) en una muestra del noroeste de México. Enseñanza e Investigación en Psicología, 16(1), 73-83.

Gerbic, P., y Stacey, E. (2005). A purposive approach to content analysis: Designing analytical frameworks. The Internet and Higher Education, 8(1), 45-59. https://doi.org/10.1016/j.iheduc.2004.12.003

Gifre, M., y Esteban, M. (2012). Consideraciones educativas de la perspectiva ecológica de Urie $\begin{array}{llll}\text { Bronfenbrenner. Contextos } \quad \text { Educativos, } & \text { 79-92. }\end{array}$ http://dialnet.unirioja.es/servlet/articulo?codigo $=3972894$

Gil, J. (2016). Variables asociadas a la autoeficacia percibida por el profesorado de ciencias en educación secundaria. Revista de Educación, (373), 85-108. https://doi.org/10.4438/1988-592X-RE-2016-373-322

González-Arratia, N., López, J., Valdez, J., Oudhof, H. y González, S. (2012). Resiliencia y factores protectores en menores infractores y en situación de calle. Psicología y Salud, $22(1), 49-62$.

Gordon, K. (2001). Resilient students' goals and motivation. Journal of Adolescence, 24, 461472. https://doi.org/10.1006/jado.2001.0383

Greco, C., Morelato, G. y Ison, M. (2007). Emociones positivas: una herramienta psicológica para promocionar el proceso de resiliencia infantil. Psicodebate, 7, 81-94.

Hernández-Sampieri, R., Fernández, C. y Baptista, P. (2010). Metodología de la investigación. MacGraw Hill.

Hershkovitz, A. (2018). The student-teacher relationship in the one-to-one computing classroom. Páginas de Educación, 11(1), 37-65. 
Hidalgo, N., Martínez-Garrido, C. y Perines, H. (2018). ¿Los futuros docentes están comprometidos con la justicia social? Validación y aplicación de la traducción al castellano de la escala de justicia social. Revista Páginas de Educación, 11(2), 85-107.

Howard, S. y Johnson, B. (2000). What Makes the Difference? Children and teachers talk about resilient outcomes for children "at risk." Educational Studies, 26(3), 321-337. https://doi.org/10.1080/03055690050137132

Landa, M. y Ramírez, M. (2018). Diseño de un cuestionario de satisfacción de estudiantes para un curso de nivel profesional bajo el modelo de aprendizaje invertido. Páginas de Educación, 11(2), 153-175. https://doi.org/10.22235/pe.v11i2.1632

Leonard, J. (2011). Using Bronfenbrenner's Ecological Theory to Understand Community Partnerships: A Historical Case Study of One Urban High School. Urban Education, 46(5), 987-1010. https://doi.org/10.1177/0042085911400337

López, V. (2010). Educación y resiliencia: alas de la transformación social. Actualidades Investigativas en Educación, 10(2), 1-14.

Martín-Lagos, M. (2018). Educación y desigualdad: una metasíntesis tras el 50 aniversario del Informe Coleman. Revista de Educación, (380), 186-209.

Martínez-abad, F., Bielba-calvo, M. y Herrera-García, M. (2017). Evaluación, formación e innovación en competencias informacionales para profesores y estudiantes de Educación Secundaria. Revista de Educación, (376), 110-134. https://doi.org/10.4438/1988592X-RE-2017-376-346

Ministerio de Educación de Chile (Mineduc). (2012). Informe Técnico Simce 2012. Agencia de Calidad de la Educación. Santiago de Chile. https://s3-us-west2.amazonaws.com/documentos-web/ Informe_Tecnico_Simce_2012.pdf

Ministerio de Educación de Chile (Mineduc). (2013a). Ministerio de Educación de Chile, Decreto 129. Santiago de Chile: Biblioteca del Congreso Nacional. https://doi.org/10.1145/1542130.1542154

Ministerio de Educación de Chile (Mineduc). (2013b). Nuevos Estándares Aprendizaje. Santiago de Chile. https://www.curriculumnacional.cl/614/articles-207508_estandar.pdf

Muñoz, V. y De Pedro Sotelo, F. (2005). Educar para la resiliencia. Un cambio de mirada en la prevención de situaciones de riesgo social. Revista Complutense de Educación, 16(1), 107-124.

Pitzer, J. y Skinner, E. (2016). Predictors of changes in students' motivational resilience over the school year: The roles of teacher support, self-appraisals, and emotional reactivity. International Journal of Behavioral Development, 41(1), 15-29. https://doi.org/10.1177/0165025416642051

Ramírez-Granizo, I., y Castro-Sánchez, M. (2018). Análisis de los niveles de resiliencia en función del género y factores del ámbito educativo en escolares. Education, Sport, Health and Physical Activity, 2(1), 50-61.

Reoyo, N., Carbonero, M. y Martín, L. (2017). Características de eficacia docente desde las perspectivas del profesorado y futuro profesorado de secundaria. Revista de Educación, (376), 62-86. https://doi.org/10.4438/1988-592X-RE-2017-376-344

Rodríguez-Fernández, A., Ramos-Díaz, E., Ros, I., Fernández-Zabala, A. y Revuelta, L. (2016a). Bienestar subjetivo en la adolescencia: el papel de la resiliencia, el autoconcepto y el 
apoyo social percibido. Suma Psicológica, 23(1), 60-69. https://doi.org/10.1016/j.sumpsi.2016.02.002

Rodríguez-Fernández, A., Ramos-Díaz, E., Ros, I., Fernández-Zabala, A., y Revuelta, L. (2016b). Resiliencia e implicación escolar en función del sexo y del nivel educativo en educación Aula Abierta, 44(2), 77-82. https://doi.org/10.1016/j.aula.2015.09.001

Rodríguez-Fernández, A., Ramos-Díaz, E., Ros, I., y Zuazagoitia, A. (2018). Implicación escolar de estudiantes de secundaria: la influencia de la resiliencia, el autoconcepto y el apoyo $\begin{array}{lllll}\text { social } \quad \text { percibido. } \quad \text { Educación } & \text { 87-108. }\end{array}$ https://dialnet.unirioja.es/servlet/articulo?codigo $=6266704$

Salvo-Garrido, S., y Cisternas-Salcedo, P. (2020). Compromiso e influencia del líder directivo desde la resiliencia socioeducativa. Revista Electrónica de Investigación Educativa, 22.

Salvo-Garrido, S., Miranda, H., Vivallo, O., Gálvez-Nieto, J., y Miranda-Zapata, E. (2020). Estudiantes resilientes en el área de matemática: examinando los factores protectores y de riesgo en un país emergente. Revista Iberoamericana de Diagnóstico y Evaluación - e Avaliação Psicológica, RIDEP, 2(55), 43-57. https://doi.org/10.21865/RIDEP55.2.04

Salvo-Garrido, S., Miranda, H., Vivallo, O., Gálvez-Nieto, J. y Miranda-Zapata, E. (2019, diciembre). Students with Socioeconomic Disadvantages who have Academic Success in Language: Examining Academic Resilience in South America. Preprints, 1-11. https://doi.org/10.20944/preprints201912.0043.v1

Salvo, S., Bravo-Sanzana, M., Miranda-Vargas, H., Forés, A. y Mieres-Chacantana, M. (2017). ¿La promoción de la resiliencia en la escuela puede contribuir con la política pública de salud? Salud Pública de México, pp. 214-215.

Sandoval-Hernández, A. y Białowolski, P. (2016). Factors and conditions promoting academic resilience: a TIMSS-based analysis of five Asian education systems. Asia Pacific Education Review, 17(3), 511-520. https://doi.org/10.1007/s12564-016-9447-4

Schettini, P. y Cortazzo, I. (Eds.). (2015). Análisis de datos cualitativos en investigación social. EdulP (Primera).

Semanchin, A., Bowen, E. y Ball, A. (2018). "School definitely failed me, the system failed me": Identifying opportunities to impact educational outcomes for homeless and child welfareinvolved youth. Children and Youth Services Review, 91, 66-76. https://doi.org/10.1016/j.childyouth.2018.05.032

Shirley, D., Fernández, M., Ossa, M., Berger, A., y Borba, G. (2013). La Cuarta Vía de liderazgo y cambio en América Latina: perspectivas en Chile, Colombia y Brasil. Pensamiento Educativo. Revista de Investigación Educacional Latinoamericana, 50(2), 5-27. https://doi.org/10.7764/PEL.50.2.2013.2

Sointu, E., Savolainen, H., Lappalianen, K. y Lambert, M. (2016). Longitudinal associations of student-teacher relationships and behavioural and emotional strengths on academic achievement. Educational Psychology, 1-11. https://doi.org/10.1080/01443410.2016.1165796

Spellman, K. (2015). Educating for resilience in the North: Building a toolbox for teachers. Ecology and Society, 20(1). https://doi.org/10.5751/ES-07243-200146

Swanson, J., Valiente, C., Lemery-Chalfant, K. y O’Brien, T. (2011). Predicting early adolescents' academic achievement, social competence, and physical health from 
parenting, ego resilience, and engagement coping. Journal of Early Adolescence, 31(4), 548-576. https://doi.org/10.1177/0272431610366249

Toland, J. y Carrigan, D. (2011). Educational psychology and resilience: New concept, new opportunities. School Psychology International, 32(1), 95-106. https://doi.org/10.1177/0143034310397284

Turner, G. (2017). Building resilience in school nurses. British Journal of School Nursing, 12. https://doi.org/10.12968/bjsn.2017.12.2.98

Uriarte, J. (2005). La resiliencia. Una nueva perspectiva en psicopatología del desarrollo. Revista de Psicodidáctica, 10(2), 61-79. https://www.redalyc.org/pdf/175/17510206.pdf

Velásquez, G. y Aguayo, P. (2011). Factores protectores resilientes de los estudiantes de la Licenciatura en Ciencias de la Educación de la UATx. En C. M. de Comie e I. Educativa (eds.), xI Congreso Nacional de Investigación Educativa/16. Sujetos de la Educación (pp. 1-9). Monterrey, Nuevo León, México.

Vieytes, R. (2004). Metodología de la investigación en organizaciones, mercado y sociedad: epistemología y técnicas. De Las Ciencias.

Wu, G., Feder, A., Cohen, H., Kim, J. J., Calderon, S., Charney, D. S. y Mathé, A. A. (2013). Understanding resilience. Frontiers in Behavioral Neuroscience, 7(10), 1-15. https://doi.org/10.3389/fnbeh.2013.00010

Yu, X., Lau, J. T. F., Winnie W. S., M., Zhang, J., Lui, W. W. S. y Zhang, J. (2011). Factor structure and psychometric properties of the connor-davidson resilience scale among chinese adolescents. Comprehensive Psychiatry, 52(2), 218-224. https://doi.org/10.1016/j.comppsych.2010.05.010 